\title{
Cross Layer based Congestion Control Scheme for Mobile Ad hoc Networks
}

\author{
S.Sheeja \\ Research Scholar \\ Bharathiar University \\ Coimbatore
}

\author{
Ramachandra V.Pujeri, PhD. \\ Vice Principal \\ KGiSL Institute of Technology, Saravanampatti, \\ Coimbatore
}

\begin{abstract}
Mobile Ad Hoc Networks consists of mobile nodes which are organized in a random manner. It can communicate with each other without any centralized infrastructure. Due to congestion, the packet loss is heavily occurred in the particular link. In order to avoid congestion, cross layer based congestion control scheme is proposed for reducing the packet losses in the network. The proposed scheme contains four phases. In first phase, the cross layer design is proposed to ensure that the information sharing can be done between the different layers in protocol stack. In second phase, the congestion detection scheme is explored which attains packet loss rate and congestion scale factor. In third phase, congestion control is achieved using cross layer approach. Here the congestion route is determined based on the path gain, buffer tenancy fraction. In fourth, new packet format is proposed. Each node maintains the congestion scale value, buffer tenancy fractional value. By extensive simulation, the proposed scheme achieves better throughput, congestion ratio, packet delivery ratio, low end to end delay and overhead than the existing schemes.
\end{abstract}

\section{Keywords}

Congestion Scale factor, MANET, Buffer tenancy fraction, cross layer, queue length, mobility, speed, packet delivery ratio, throughput, end to end delay and overhead.

\section{INTRODUCTION}

\subsection{Mobile Ad hoc Networks (MANETs)}

Mobile Ad hoc Networks (MANETs) are the collection of mobile nodes without any centralized infrastructure. Due to the presence of high mobility of the nodes, the packet loss occurs frequently in the network. So the network congestion may be induced at any time. This will lead to lack of communication, lack of support, and more overhead. The main characteristics of MANET are dynamic topology, no access point, energy constrained, battery constrained. Mobile ad hoc networks used in various potential applications like earthquake, search and rescue operations, etc.

\subsection{Congestion Control in MANETs}

The main issue in MANET is congestion control. It is totally associated to control the incoming traffic into a telecommunication network. The solution is to send a reduced packet sending rate to overcome the effect of congestion effectively. In general, the Transmission Control Protocol mainly combines the congestion control and dependability mechanism without explicit feedback about the congestion position.

The congestion control principles include packet conservation, Additive Increase and Multiplicative Decrease (AIMD) in sending rate, stable network. The other techniques for congestion control are end system flow control, network congestion control, network based congestion avoidance, and resource allotment

The packet loss can be condensed by involving congestion control over a mobility and failure adaptive routing protocol at the network layer. The congestion causes following difficulties:

More delay: Several congestion control mechanism takes much time for detecting congestion. It leads to heavy delay. Sometimes the usage of new routes in some critical situations is advisable. The main problem is the delay moving for route searching in on-demand routing protocol.

High Overhead: Congestion control mechanism takes effort for processing and communication in new routes for discovering it. It also takes effort in multipath routing for maintaining the multi-paths, though there is another protocol.

More packet losses: If the congestion is detected, the packets may be lost. Congestion control solution is applied either by decreasing the sending rate at the sender, or dropping packets at the intermediate nodes or by both methods to decrease the traffic load. If the high packet loss rate occurs, small throughput may be occurred in the network.

\subsection{Cross Layer design}

Cross layer design [23] is said to be the violation of layered communication architecture in the protocol design with respect to the original architecture. This design emphasizes on the network performance by enabling the different layers of the communication stack to share state information or to coordinate their actions in order to jointly optimize network performance.

Distributed algorithms can exploit a cross-layer design to enable each node to perform fine-grained optimizations locally whenever it detects changes in network state. Mobility causes changes for the physical layer (for e.g. interference levels), the data link layer (for e.g. link schedules), the routing layer (for e.g. new neighbouring nodes), and the transport layer (for e.g. connection timeouts). As such, a cross-layer based design enhances the capability of the node to manage its resources in the mobile environments Antenna arrays can also enable the reception of multiple packets simultaneously on the wireless channel and the data packets corresponding to several connections could also arrive simultaneously at a node. The cooperation of various layers such as routing, data link, and physical layer can ensure the forwarding of data for all the connections within time.

\subsection{Need for Cross layer design}

Cross layer design [23] offers performance benefits for a particular system. In contrast, the architecture offers a model for sustained innovation in a system, so it offers long-term gains. The short-term performance gains of cross-layer design may be more significant for the network user to make efficient use of limited node resources.

The following issues raise the need for cross layer design: 
- Due to multipath, the response of the wireless channel varies over time and space which leads to short-term fading. These variations may be caused due to either motion of the wireless device or changes in the surrounding physical environment, and lead to errors at the receiving end. This causes bursts of errors to occur during which packets cannot be successfully transmitted on the link. Furthermore, if very strong forward error correction codes at low error rates are employed to eliminate the burst errors then it reduces the spectral efficiency.

- There are also spatial and temporal variations on a much greater timescale due to the small scale variations. Large-scale channel variation means that the average channel response depends on user locations and the level of interference on the channel. This channel variation occurs because of some users may essentially demand more channel access time than others based on their location and/or mobile velocity, even if their data rate requirement is the same as or less than other users. The improvement in the channel characteristics can be improved if the strict physical and MAC layer boundaries can be made soft with the help of cross layer design.

\section{Related Work}

Shafiullah Khan et.al [1] discussed the importance of cross layer security mechanisms and routing protocols for multi-hop wireless networks by critical comparison. The selection of optimal path for routing and the detection of multilayer security attacks cannot be achieved with the traditional approaches. They suggest that cross layer design is the only solution to cope with these kinds of challenges in multi-hop wireless networks.

In [2], the performance of four queuing disciplines like first in first out, Random early detection, priority queuing and weighted fair queuing are evaluated which is implemented in the AEERG protocol. Rajeswari et.al presented simulationbased performance evaluation and comparison of three queuing techniques for different number of nodes, packet size and pause time for the impact of using random-early drop as compared to drop-tail policy and weighted fair scheduling.

Shitalkumar Jain et.al [3] reviewed that signal strength based measurements used to improve such packet losses and no need to retransmit packets. So, the node based and link based signal strength can be measured. If a link failed due to mobility, then signal strength measurement provides temporary higher transmission power to keep link alive. When a route is likely to fail due to weak signal strength of a node, it will find alternate path. consequently avoids congestion.

G.S.Sreedhar \& Dr.A.Damodaram [4] proposed Medium Access Level Multicast Routing for Congestion Avoidance in Multicast Mobile Ad Hoc Routing Protocol to avoid the congestion in networks. This protocol derives an algorithm that transmits the data in multicast manner at group level unlike other multicast protocols, concentrating of data transmission in a sequence to every targeted node. Being independent, the proposed work was with group of either tree or mesh.
S.A.Jain [5] provides their deep insight to TCP congestion control mechanism and proposed route failure detection mechanisms.It is suggested that most of the mechanisms are aiming at increasing the life time of the networks and improving the performance of the parameters like packet delivery ratio, end to end delay etc.

Senthil Kumaran, T. and V. Sankaranarayanan [6] presented the Congestion Free Routing in Ad hoc networks (CFR), based on dynamically estimated mechanism to monitor network congestion by calculating the average queue length at the node level. While using the average queue length, the nodes congestion status divided into the three zones like safe zone, congested zone and congested zone. The scheme utilizes the non-congested neighbors and initiates route discovery mechanism to discover a congestion free route between source and destination. This path becomes a core path between source and destination. To maintain the congestion free status, the nodes which are helping data packet transmission periodically calculate their congestion status at the node level.

K. Srinivas, A. A. Chari [7] proposed the cross layered model of congestion detection an control mechanism that includes energy efficient congestion detection, Zone level Congestion Evaluation Algorithm [ZCEA] and Zone level Egress Regularization Algorithm [ZERA], which is a hierarchical cross layer based congestion detection and control model. By experimental results the proposed approach achieved the better resource utilization, energy efficiency in congestion detection and congestion control.

In [8], [9] authors introduced the concept of cross layer design and congestion detection using cross layer approach. The aim of the work is to improve the performance based on gathering the bandwidth and delay information on the link layer.

S. Subburamm and P. Sheik Abdul Khader [10] proposed AODV-EBRP mechanism to eliminate network congestion by reducing the rebroadcasting of data packets among the neighboring nodes. In addition, when a link breakage is anticipated, this mechanism will identify a new route, having free buffer space, to establish a forward link to the destination. This mechanism would ensure that routes are generated and links established through a node that has more buffer space, in order that more number of packets could be stored/forwarded through the node free of congestion.

Rajkumar, G. et.al [11] proposed congestion aware multi path routing protocol to reduce the congestion losses. In this protocol, a congestion control technique is followed which proactively detects node level and link level congestion and performs congestion control using the fault-tolerant multiple paths. The congestion detection algorithm is buffer based. On reception of a data packet, each intermediate node monitors its current buffer size and calculates a running average value using exponential weighted moving average formula. If this average value becomes greater than a predefined threshold, then the congestion is detected. Whenever the source node receives the congestion control packet sent by the congested node, it executes the congestion control algorithm. This proposed scheme enables more nodes to salvage a dropped packet.

In [12], [13],[14],[15], [16], they analysed the congestion minimization using cross layer approach, congestion avoidance and congestion control schemes. The main analysis of the work is to balance the congestion avoidance and delay 
in MANET. But they have not focussed on the link capacity determination. The basic factor of congestion occurrence is not considered in these scenarios.

V. Thilagavathe and Dr. K. Duraiswamy [17] proposed the a cross-layer based technique to overcome congestion that occurs in MAC and transport layer in MANET. The proposed technique was applied over a Ad hoc On demand Multipath Reliable and Energy Aware QoS Routing Protocol (AOMPREQR). The technique of additive increase and multiplicative decrease (AIMD) was applied for rate based congestion control of transport layer protocol. If source receives congestion status information from both MAC and transport layer simultaneously for the same route, then congestion free route can be established for transmission, without performing rate control.

Karunakaran et.al [18] presented a Cluster Based Congestion Control (CBCC) protocol that consists of scalable and distributed cluster-based mechanisms for supporting congestion control in adhoc networks. The distinctive feature of approach was that it was based on the self-organization of the network into clusters. The clusters autonomously and proactively monitor congestion within its localized scope. The protocol consists of clustering mechanism, traffic rate estimation and traffic rate adjustment. The present approach improved the responsiveness of the system when compared to end-to-end techniques. After estimating the traffic rate along a path, the sending rate of the source nodes was adjusted accordingly. Thus the protocol looked forward the injection of dynamic flows in the network and proactively adjusts the rate while waiting for congestion feedback.

Mohammad Amin Kheirandish Fard et.al [19] explored an end-to-end threshold-based algorithm which enhances congestion control to address link failure loss in MANET. It consists of two phases. First, threshold-based loss classification algorithm distinguished losses due to link failure by estimating queue usage based on Relative One-way Trip Time (ROTT). Second phase adjusted RTO for new route by comparing capabilities of new route to the broken route using available information in Transport layer such as ROTT and number of hops. Threshold-based loss classification algorithm used queue usage to classify network state periodically into congestion or non-congestion mode. Any retransmission timeout in period which MANET was non-congested mode is an indication of link failure loss. In addition, implementation showed that small percentage of three duplicate acknowledgments which emerge immediately after route recovery might be result of route changes.

Kazuya et.al [20] analyzed a routing protocol that uses multiagents to reduce network congestion for a Mobile Ad hoc NETwork (MANET). MANET is a mul- tihop wireless network in which the network components such as PC, PDA and mobile phones are mobile. The components can communicate with each other without going through a server. Two kinds of agents are engaged in routing. One is a Routing Agent that collects information about network congestion as well as link failure. The other is a Message Agent that uses this information to get to their destination nodes.

In [21], the congestion control is achieved through the low rate video streaming. The basic purpose and aim of the work is to provide congestion avoidance for multi tree overlays in the MANET. Here the distortion estimation is also calculated by estimated distortions of the each groups with estimated number of the receiving nodes in each group. So it enables the reliability on sending the packet while transmitting the video in CoDio sense.

The paper is organized as follows. The Section 1 describes with overview of congestion and its issues. Section 2 deals with the Related Work. Section 3 is devoted for the implementation of proposed algorithm. Section 4 describes the performance analysis and the last section concludes the work.

\section{Implementation of Proposed Algorithm}

The proposed cross layer congestion control scheme depends on the cross layer design, detection of congestion using cross layer approach and congestion control procedure.

\subsection{Cross Layer Design}

In MANET traditional approach, each layer of the IP protocol stack operates independently. The information is being shared between the adjacent layers only. Due to the dependencies between physical and upper layers, the traditional approach is not suitable for Mobile Ad hoc Networks. There is need to cross the normal layer boundaries to improve the performance of communication and hence better than the application layer performance. In Cross layer design, the data is shared between the different protocol layers dynamically.

So that, the information can be exchanged between any different layers of the TCP/IP protocol stack. Figure 1 illustrates the cross layer design for information sharing across different layers.

In Wireless network, physical layer, Media Access Control (MAC) layer and routing Layer is combined for network resource. At physical layer, transmission power and data rate is decided which affects MAC and routing decisions. The MAC layer is responsible for scheduling and allocating the wireless channel, it will determine the available transmitter bandwidth and the packet delay. Routing layer also depends on bandwidth and delay to select the link.

The routing layer chooses the route to send the data packets to the destination. The routing decision will change the contention level at the MAC layer and accordingly the physical layer parameters. Because of adaptation of layers end to-end performance can be optimized. Any design changes in the protocol stack when adding interaction between different layers may have effect on the whole system. So cross layer design use with caution.

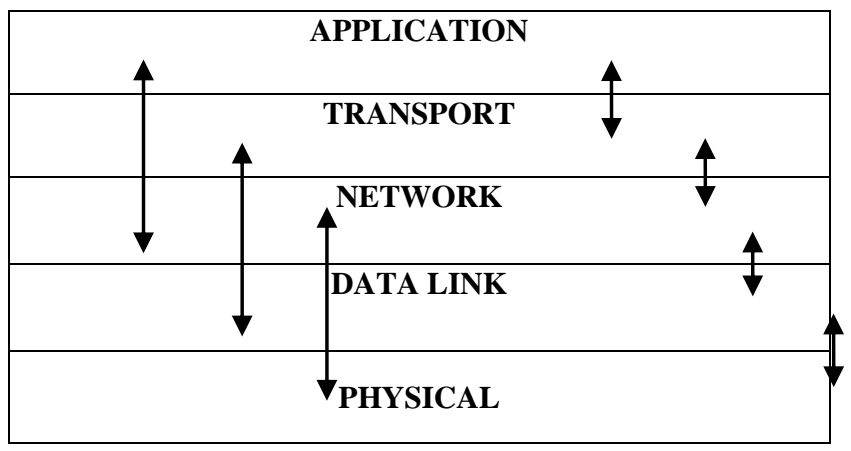

Figure1. Cross layer design - Information Sharing scheme 


\subsection{Congestion Detection using cross layer approach}

Step 1: Source node sends the data packet to destination node via intermediate nodes to establish the route discovery process.

Step 2: Set the interval $T_{n}$ between the two adjacent packets in MAC layer. $t_{s}$ is the arrival time of first data packet. $t_{d}$ is the arrival time of last data packet. $\mathrm{T}_{\mathrm{k}}$ is the average processing time of data packet in a local node.

Step 3: $t_{\mathrm{e}}$ is the transmission time of data packets, and $\mathrm{q}$ is an adjustable parameter, which is set to 0.7 . The value of $T_{k}$ is updated when a data packet is sent out.

Step 4: Calculate the packet loss rate. It is given by

$$
P_{L R}\left(t_{1}, t_{2}\right)=\frac{\int_{t_{1}}^{t_{2}} 1_{\left\{G(t)=D_{l}\right\}} d F(t)}{\int_{t_{1}}^{t_{2}} d F(t)}
$$

where $\mathrm{F}(t)$ is the arrival process for user packets. Here, the denominator represents the number of user packets sent in $\left(t_{1}\right.$, $t_{2}$ ) and the numerator represents the number of lost user packets.

Step 5: Calculate the congestion scale factor

The Value of $\mathrm{C}_{s f}$ is given as follows,

$$
\begin{aligned}
& C_{s f}=\frac{T_{n}}{T_{k}} \\
& \mathrm{~T}_{\mathrm{n}}=(1-\mathrm{q}) \times \mathrm{T}_{\mathrm{n}}+\mathrm{q} \times\left(\mathrm{t}_{\mathrm{s}}-\mathrm{t}_{\mathrm{d}}\right)-P_{L R}\left(t_{1}, t_{2}\right) \\
& \mathrm{T}_{\mathrm{k}}=(1-\mathrm{q}) \times\left(\mathrm{t}_{\mathrm{s}}-\mathrm{t}_{\mathrm{d}}\right)+\mathrm{q}_{\mathrm{e} \mathrm{t}}-P_{L R}\left(t_{1}, t_{2}\right)
\end{aligned}
$$

If $C_{s f}>1$, the arrival rate $\mathrm{f}$ the data packets is bigger than the departure rate of data packets, which indicates congestion may possibly happen in the future route.

Step 6: Let the threshold of buffer tenancy fraction be $B_{t t f}$. If $B t f>B_{t t f}$, indicating that congestion is happened. Based on the buffer queue length in local node, the congestion can be detected. If the number of data packets in buffer queue exceeds the threshold value, indicating that the data packets will overflow the buffer queue in short time and congestion will happen in the local mobile node.

\subsection{Congestion Control Procedure}

Let the signal to interference to noise ratio (S) of the link $l$ is given by

$$
S=\frac{P_{k} G_{d}}{\sum_{d \neq k} P_{o} G_{k o}+n_{k}{ }^{\prime}}
$$

where $\mathrm{P}_{\mathrm{k}}$ is the transmission power on the link $k, G_{d}$ is the path gain of the link $k, G_{k o}$ is the path gain of the node on link $\mathrm{k}$ to another node on the link $o . \mathrm{n}_{\mathrm{k}}$ is the thermal noise on the link $k$. The maximum capacity attainable in the link $k$ is determined using Shannon capacity theorem.

$$
C_{l}=\frac{1}{T} \log (1+M . S) p k t s / \mathrm{sec}
$$

Where $\mathrm{T}$ is the symbol period and $\mathrm{M}$ is the constant based on modulation scheme used.
Step 1: Set the initial transmission rate $x_{i}=x_{\text {initial }}$.

Step 2: Set power of the link $\mathrm{k}$ is $\mathrm{P}_{\mathrm{k}}=\mathrm{P}_{\mathrm{k} \text {,min }}$

Step 3: Broadcast the $\mathrm{S}$ to the entire link.

Step 4: Update path gain of the link $\left(\mathrm{G}_{\mathrm{d}}, \mathrm{G}_{\mathrm{ko}}\right)$ after receiving the broadcasting signals.

Step 5: Calculate the maximum capacity of the link $\mathrm{C}_{l}$. Step 6: Determine the loss probability

$$
\gamma_{m}(t)=\left\{\begin{array}{cc}
\frac{\max \left(0,\left(x_{l}(t)-c_{l}(t)\right)\right)}{x_{l}(t)} & x_{l}>0 \\
0 & x_{l}=0
\end{array}\right.
$$

Step 7: Calculate the data sent to the link $k$,

$$
m_{k}(t)=\frac{\gamma_{m}(t) * S}{P_{k} G_{k k}}
$$

Step 8: Set the threshold value of path gain G, Congestion Scale Factor and Buffer tenancy fraction.

Step 9: If the any parameter below its threshold value, the route is considered to be a congestionless route. Otherwise

Route is considered as congestion based route.

Step 10: Choose the alternative path while congested route found.

\subsection{Proposed Packet Format}

\begin{tabular}{|l|l|l|l|l|l|l|}
\hline $\begin{array}{l}\text { Source } \\
\text { ID }\end{array}$ & $\begin{array}{l}\text { Dest. } \\
\text { ID }\end{array}$ & $\begin{array}{l}\text { Hop } \\
\text { Count }\end{array}$ & MAC & C $_{\mathrm{sf}}$ & $\mathrm{P}_{\mathrm{LR}}$ & $\mathrm{B}_{\mathrm{tf}}$ \\
\hline
\end{tabular}

Figure 2. Proposed packet format

- The proposed packet is shown in figure 2. It contains source id, destination id occupies 2 bytes. Source id is used to identify the node which is ready to discover the route. Destination node verifies the packet received from route which contains source id.

- The Hop count is incremented once the packet is successfully sent. It occupies 1 byte field. Hop count determines number of nodes is connected to the particular node.

- Medium Access Control (MAC) is for accessing the particular channel.

- Congestion scale fraction is used to verify the congestion will occur or not. It occupies 4 byte field.

- $\quad \mathrm{P}_{\mathrm{LR}}$ is the packet loss rate which occupies 2 bytes field.

- $\quad B_{t f}$ is used to verify the occupation of the buffer which occupies 2 bytes field.

\section{Performance Analysis}

Network Simulator (NS 2.34) is used to simulate the proposed algorithm. In this simulation tool, the $\mathrm{C}++$ language is back end language and tool command language (tcl) is front end language. The basic advantage of this tool is more updation. In our simulation, 1500 mobile nodes move in a 1500 meter $\mathrm{x} 1500$ meter square region for 120 seconds simulation time. All nodes have the same transmission range of 400 meters. The simulated traffic is Constant Bit Rate 
(CBR). The simulation settings and parameters are summarized in table 1.

Table1. Simulation settings and parameters

\begin{tabular}{|l|l|}
\hline No. of Nodes & 1500 \\
\hline Area Size & 1500 X 1500 \\
\hline Mac & 802.11 \\
\hline Radio Range & $400 \mathrm{~m}$ \\
\hline Simulation Time & $120 \mathrm{sec}$ \\
\hline Traffic Source & CBR \\
\hline Packet Size & 512 bytes \\
\hline Mobility Model & Random Way Point \\
\hline Protocol & AODV \\
\hline Pause time & $4 \quad$ msec \\
\hline
\end{tabular}

\subsection{Performance Metrics}

It is evaluated mainly the performance according to the following metrics.

Control overhead: The control overhead is defined as the total number of routing control packets normalized by the total number of received data packets.

End-to-end delay: The end-to-end-delay is averaged over all surviving data packets from the sources to the destinations.

Packet Delivery Ratio: It is the ratio of the number .of packets received successfully and the total number of packets transmitted.

Throughput: It is defined as the number of packet received at a particular point of time.

The simulation results are presented in the coming part. The CLCCS is compared with ECAS [22] and CoDio ABCD protocol [21] in presence of congestion environment.

Figure 3 illustrates the topology of the proposed scheme. In existing work, it is used that the whole network to implement the congestion less route. Here, it is chosen only on the single route to achieve the congestion less routing efficiency with the help of cross layer design. The route $R_{1}$ consists of 30 mobile nodes. The mobility of these mobile nodes is relatively high. Due to mobility presence, the congestion occurs indefinitely. To overcome this problem, the proposed approach based on cross layer design proposes the congestion less route. Source node chooses the destination node based on the efficiency of the node, capacity of the link and shortest path route. Figure 4 shows that traffic creation among the nodes. To identify the packet loss, the constant bit rate traffic is implemented. The delay is produced in packet from source node to destination node via neighbor nodes. Source may choose the different paths to achieve the high packet delivery fraction.

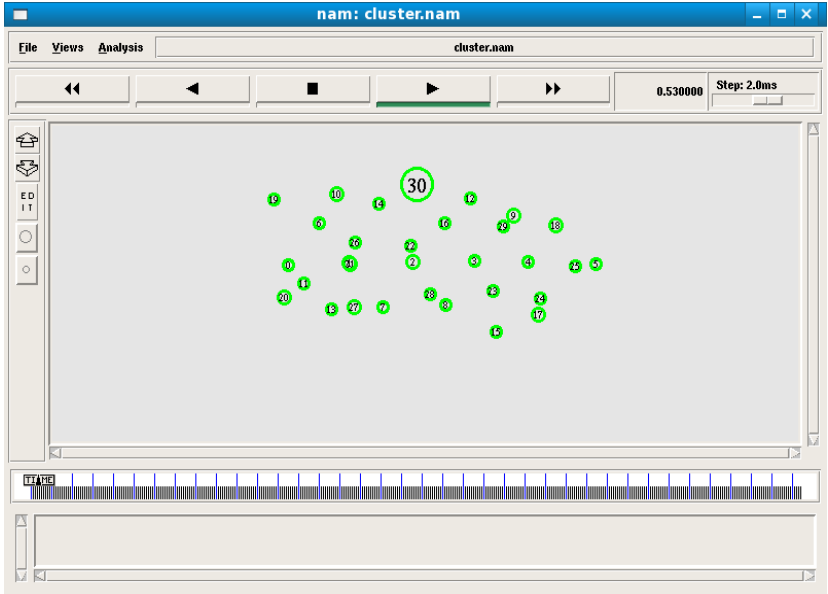

Figure3. Network topology

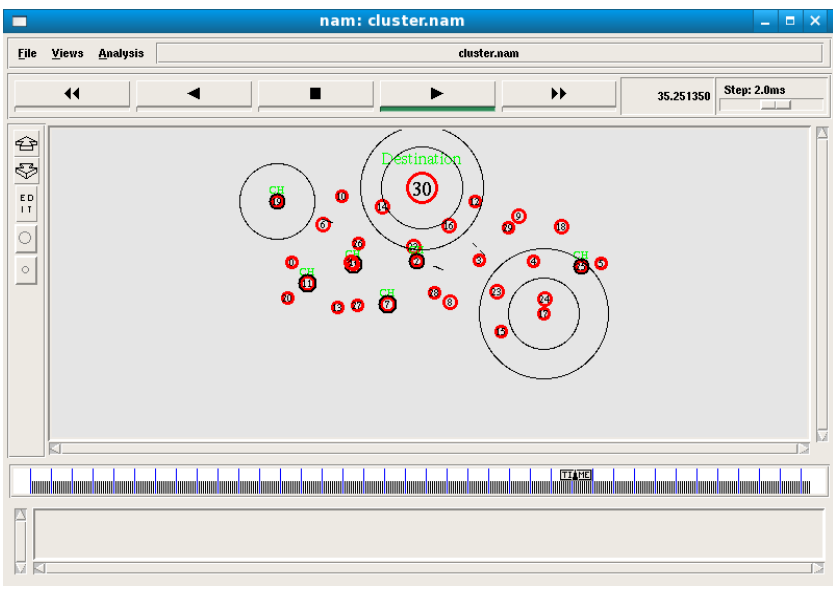

Figure 4. Traffic creation among the mobile nodes

Figure 5 shows the results of congestion ratio for varying the time from 10 to 50. From the results, it is seen that scheme CLCCS has lower congestion ratio than the ECAS and CoDio $\mathrm{ABCD}$ protocol because of congestion aware routing.

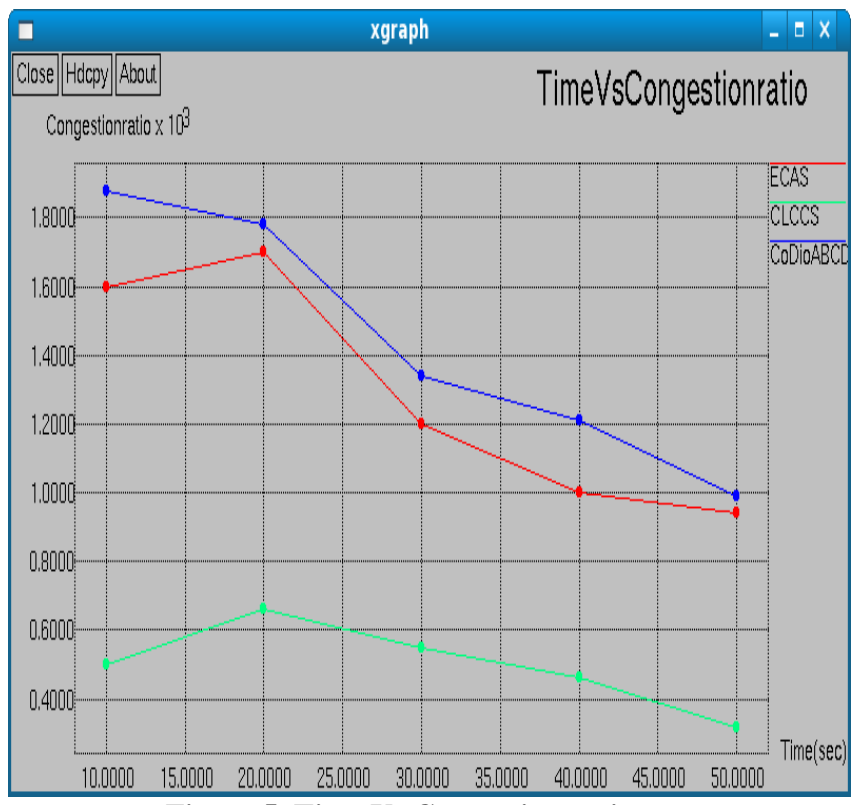

Figure 5. Time Vs Congestion ratio 
Figure 6 shows the results of packet delivery ratio for varying the throughput from 10 to 50 . From the results, it is seen that CLCCS scheme has higher delivery ratio than ECAS and CoDio ABCD protocol because of reduced congestion routing.

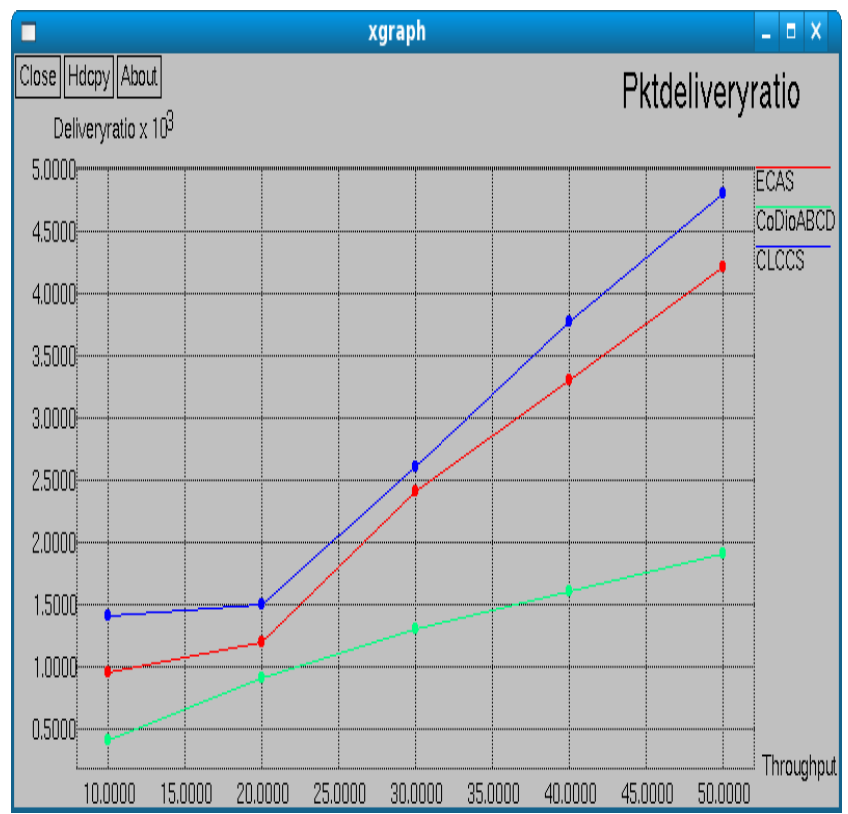

Figure 6. Throughput Vs delivery ratio

Figure 7, presents the comparison of overhead and mobility. It is clearly shown that the overhead of CLCCS has low overhead than the ECAS and CoDio ABCD protocol.

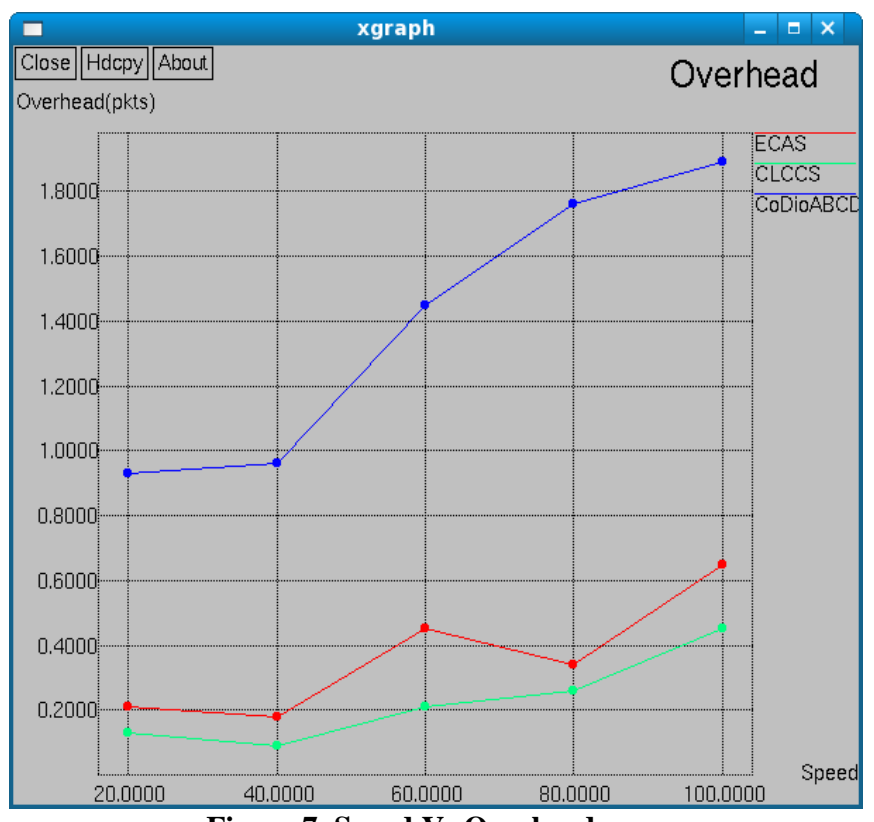

Figure 7. Speed Vs Overhead

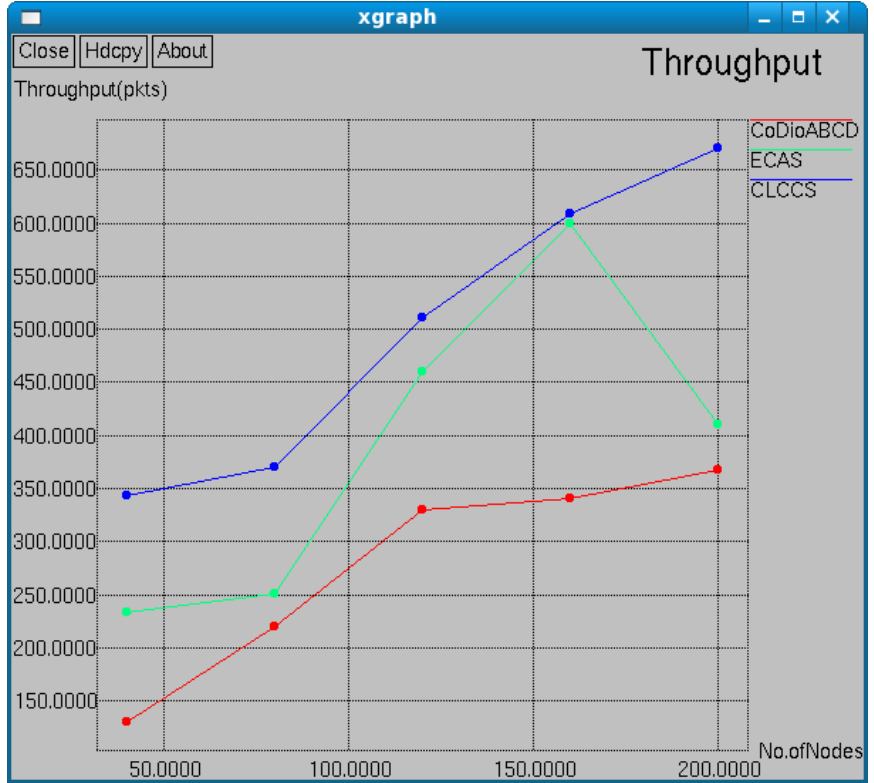

Figure 8. No.of nodes Vs Throughput

Figure 8 shows the results of No.of Nodes Vs Throughput. From the results, it is seen that CLCCSscheme has higher throughput than ECAS and CoDio ABCD protocol while varying the number of nodes from 10 to 200.

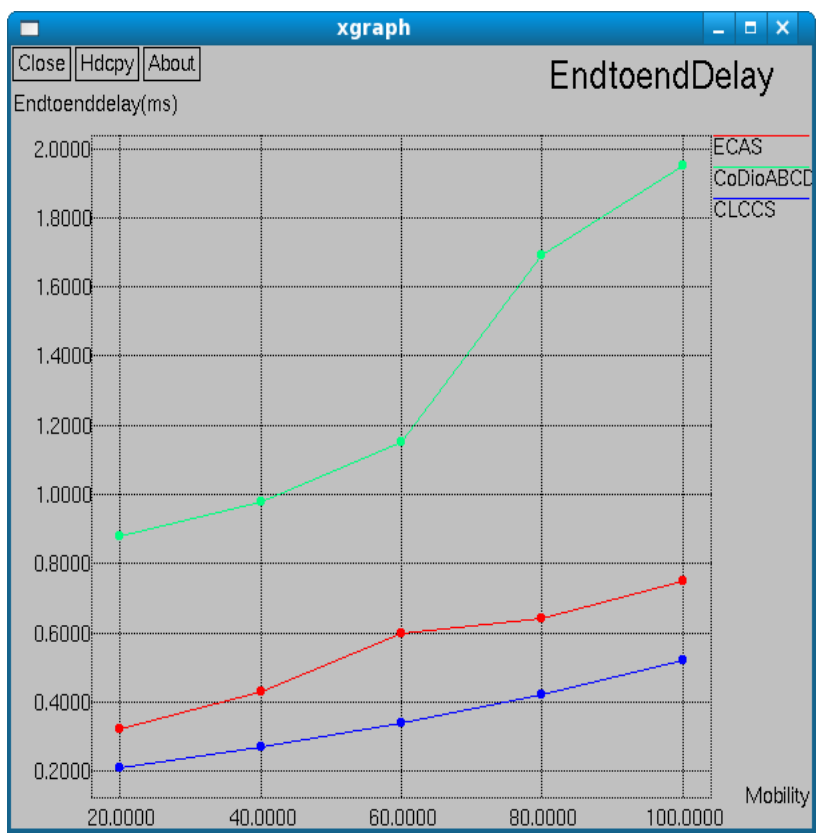

Figure 9. Mobility Vs End to End delay

Fig. 9, presents the comparison of delay while varying the mobility from 20 to 100 . It is clearly shown that the delay of CLCCS has low than ECAS and CoDio ABCD protocol. 
Table 2. Analysis of Proposed Method (CLCCS) and Existing Methods (ECAS and CoDioABCD) in terms of different parameters

\begin{tabular}{|l|l|l|l|}
\hline Metrics & CLCCS & ECAS & CoDioABCD \\
\hline $\begin{array}{l}\text { Congestion ratio } \\
(\%)\end{array}$ & $500-354$ & $1600-987$ & $1830-1000$ \\
\hline $\begin{array}{l}\text { Packet Delivery } \\
\text { Ratio (pkts) }\end{array}$ & $1494-4845$ & $1000-4127$ & $490-1945$ \\
\hline Throughput (pkts) & $348-674$ & $246-412$ & $145-362$ \\
\hline $\begin{array}{l}\text { End to end delay } \\
\text { (msec) }\end{array}$ & $0.212-0.543$ & $\begin{array}{l}0.356- \\
0.789\end{array}$ & $0.908-1.967$ \\
\hline Overhead (pkts) & $0.189-0.432$ & $\begin{array}{l}0.204- \\
0.623\end{array}$ & $0.967-1.874$ \\
\hline
\end{tabular}

\section{Conclusion}

Mobile nodes are moving randomly without any centralized administration. Due to presence of congestion, the packet loss occurs unnecessarily. So the node has to retransmit the packet. It leads to maximum energy consumption. In this research work, a Cross Layer based congestion control scheme is developed which attains congestion detection and congestion control among nodes. In the first phase of the scheme, cross layer design is proposed. Here, information is shared between the different layers of the protocol stack. In congestion detection phase, the packet loss rate is determined which ensures the detection of congestion in particular link. In congestion control phase, the route is found without congestion. Here the threshold value of path gain, congestion scales value and buffer tenancy value. By using the extensive simulation results, the proposed scheme CLCCS achieves the better throughput, packet delivery ratio, low delay and overhead than the existing schemes ECAS, CoDio ABCD protocol while varying the mobility, speed, throughput, time and number of nodes.

\section{REFERENCES}

[1] Shafiullah Khan and Kok Keong Loo, Zia Ud Din ," Cross Layer Design for Routing and Security in Multihop Wireless Networks", Journal of Information Assurance and Security, Vol.4 , pp.170-173, 2009.

[2] S.Rajeswari \& Dr.Y.Venkataramani, "Congestion Control and QOS Improvement for AEERG protocol in MANET", International Journal on Ad Hoc Networking Systems (IJANS) Vol. 2, No. 1, pp.13-21, 2012.

[3] Prof. Shitalkumar Jain, Miss.Sunita I. Usturge, "Signal Strength Based Congestion Control in MANET", Advances in Physics Theories and Applications, Vol 1, pp.26-36, 2011.

[4] G.S.Sreedhar \& Dr.A.Damodaram, “ MALMR: Medium Access Level Multicast Routing for Congestion Avoidance in Multicast Mobile Ad Hoc Routing Protocol”, Volume 12 Issue 13, pp.22-30, 2012.

[5] S.A.Jain and Sujata K.Tapkir, "A Review of Improvement in TCP congestion Control Using Route
Failure Detection in MANET", Network and Complex Systems, Vol 2, No.2, pp.9-13, 2012.

[6] Senthil Kumaran, T. and V. Sankaranarayanan, "Congestion Free Routing in Adhoc Networks", Journal of Computer Science, Vol.8, No.6, pp.971-977, 2012.

[7] K. Srinivas, A. A. Chari, “ ECDC: Energy Efficient Cross Layered Congestion Detection and Control Routing Protocol", International Journal of Soft Computing and Engineering (IJSCE) ISSN: 2231-2307, Volume-2, Issue-2, pp.316-322, 2012.

[8] Dzmitry Kliazovich, Fabrizio Granelli, "Cross-layer congestion control in ad hoc wireless networks", Elsevier, Ad Hoc Networks, Vol. 4, pp. 687-708, 2006.

[9] Marco Conti, Gaia Maselli, Giovanni, Turi \& Silvia Giordano, "Cross-Layering in Mobile Ad Hoc Network Design”, IEEE Computer Society, pp.48-51, 2004.

[10] S. Subburamm and P. Sheik Abdul Khader, "Efficient Broadcasting using Preventive Congestion Mechanism in Mobile ad Hoc Network", European Journal of Scientific Research, Vol.83, No.2, pp.302-313, 2012.

[11] Rajkumar, G. and K. Duraiswamy, "A Fault Tolerant Congestion Aware Routing Protocol for Mobile Ad hoc Networks", Journal of Computer Science, Vol.8, No.5, pp.673-680, 2012.

[12] Xiaojun Lin, and Ness B. Shroff," The Impact of Imperfect Scheduling on Cross-Layer Congestion Control in Wireless Networks", IEEE/ACM Transactions On Networking, Vol. 14, No. 2, pp.302-313, 2006.

[13] T.Suryaprakash Reddy and Dr.P.Chenna Reddy , “ OCC: Ordered congestion control with cross layer support in MANET routing", International Journal of Advanced Computer Science and Applications, Vol. 3, No. 3, pp.94-101, 2012.

[14] Prof.K.Srinivas and Prof.A.A.Chari "Cross Layer Congestion Control in MANETs and Current State of Art", International Journal of Computer Applications (0975 - 8887) Volume 29- No.6, pp.28-35, 2011.

[15] Seema Aarya, “ Congestion Minimization through Collision Detection in TDMA and CSMA/CA Scheme in Wireless Mobile Ad-Hoc Network", International Journal of Computer Applications (0975 - 8887) Volume 45- No.1, pp.14-18, May 2012,

[16] W.R. Salem Jeyaseelan and Dr. S. Hariharan, "Study on Congestion Avoidance in MANET", IJCA Special Issue on "Network Security and Cryptography" NSC, pp.7-10, 2011.

[17] V. Thilagavathe and Dr. K. Duraiswamy, "Cross Layer based Congestion Control Technique for Reliable and Energy Aware Routing in MANET", International Journal of Computer Applications (0975 - 8887), Volume 36- No.12, pp.1-6, December 2011.

[18] S.Karunakaran \& P.Thangaraj, "A Cluster Based Congestion Control Protocol For Mobile Ad hoc Networks", International Journal of Information Technology and Knowledge Management, Volume 2, No. 2, pp. 471-474, 2010.

[19] Mohammad Amin Kheirandish Fard, Sasan Karamizadeh, , Mohammad Aflaki, "Enhancing Congestion Control To Address Link Failure Loss over 
Mobile Ad-Hoc Network", International Journal of Computer Networks \& Communications (IJCNC) Vol.3, No.5, pp.177-192, Sep 2011.

[20] Kazuya Nishimura and Kazuko Takahashi, "A MultiAgent Routing Protocol with Congestion Control for MANET", European Conference on Modelling and Simulation, pp.1-6, 2007.

[21] Claudio Greco, Marco Cagnazzo and Béatrice PesquetPopescu, "Low-Latency Video Streaming With Congestion Control in Mobile Ad-Hoc Networks", IEEE Transactions On Multimedia, Vol. 14, No. 4, pp.13371350, 2012.

[22] S.Sheeja and Ramachandra.V.Pujeri, "Effective Congestion Avoidance Scheme for Mobile Ad Hoc Networks", International Journal of Computer Network and Information Security, Vol. 05, No. 1, pp.33-40, January 2013.

[23] Sandeep Sharma, Rajesh Mishra And Karan Singh, "Current trends and future aspects in Cross layer Design for the Wireless Networks", Computer Science \& Information Technology (CS \& IT), pp. 283-296, 2012.

\section{AUTHORS' PROFILE}

S.Sheeja, Received B.Sc in Electronics from V.L.B Janaki Ammal College of Arts and Science, Coimbatore, MCA degree from Bharathiar University, M.Phil degree in
Computer Science from Bharathiar University, Coimbatore in 2000, 2003 and 2007 respectively. She has 9 years of teaching experience. She is currently pursuing doctoral research in Computer Networks in Bharathiar University. Her primary research interests are related to Computer Networks. Currently, she is working as Assist Professor in Computer Applications Department at Karpagam University, Coimbatore.

Dr.Ramachandra.V.Pujeri, Received the B E in Electronics and Communication Engineering from Karnataka University, Dharwad, ME in Computer Science and Engg from PSG College of Technology, Coimbatore, Ph.D in Information and Communication Engineering from Anna University, Chennai, MBA in Human Resource Management, from Pondicherry University, Pondicherry, in 1996, 2002, 2007 and 2008 respectively. He is active life member of ISTE, SSI, MIE, ACS and IEE. He has written three textbooks. He is having around 18 years of teaching experience in the various top ten engineering colleges in India. $\mathrm{He}$ is an active expert committee member of AICTE, NBA, DoEACC, NACC and various Universities in India. Currently, under him ten research scholars pursuing their Ph.D. His research interests lie in the areas of Computer Networking, Operating System, Software Engineering, Software Reliability, Modeling and Simulation, Quality of Services and Data Mining. Currently, he is working as Vice-Principal of KGiSL Institute of Technology, Coimbatore. 